\title{
Hepatopulmonary syndrome associated with iron overload in sickle cell anaemia
}

\section{To the Editors:}

At present, it is estimated that 10,000 to 20,000 patients in the USA are treated with long-term blood transfusion [1], including patients with sickle cell disease (SCD) who are often transfused to prevent stroke [2,3]. Iron overload is common in patients with recurrent transfusion, and the degree of iron overload may correlate with the rate of transfusions [4, 5]. While iron overload and cirrhosis have not been definitively linked in patients with SCD, $>11 \%$ of patients with SCD were found to have cirrhosis on autopsy and a possible link has long been suggested [6].

Hepatopulmonary syndrome (HPS) is a common pulmonary complication of cirrhosis, occurring in $5-32 \%$ of patients with cirrhosis [7]. HPS is pathologically characterised by the formation of dilated pulmonary capillaries or, less often, microscopic pleural and pulmonary arteriovenous communications [8]. Clinically, it is characterised by progressive hypoxaemia that often reverses following liver transplantation [6].

Patients with SCD are predisposed to complications of the pulmonary parenchyma and pulmonary vasculature, which often lead to chronic hypoxaemia. Well-described complications include pulmonary arterial hypertension, pulmonary venous hypertension, fat emboli, acute chest syndrome and pulmonary fibrosis [9]. However, to our knowledge, HPS associated with iron overload from SCD has not been described. In this article, we describe two patients presenting with unexplained hypoxaemia who were found to have HPS in association with SCD.

The first patient was a 40-yr-old female with haemoglobin SS disease (sickle cell anaemia) who had required long-term transfusion. She averaged eight to 10 hospitalisations each year due to acute pain crises, and she typically received multiple transfusions during each admission. Her haemoglobin was $7 \mathrm{~g} \cdot \mathrm{dL}^{-1}$ at baseline, but often decreased to $\leqslant 5 \mathrm{~g} \cdot \mathrm{dL}^{-1}$ during acute pain crises. She presented for pulmonary outpatient consultation with dyspnoea and hypoxaemia. Her oxygen saturation was $92 \%$ at rest and $82 \%$ with ambulation, with a resting alveolar-arterial gradient of $32 \mathrm{mmHg}$. Her examination revealed icteric sclera, a $2 / 6$ systolic murmur at the cardiac apex, normal chest auscultation, no clubbing and no spider angiomata. Initial testing included a chest radiograph, which did not reveal any pulmonary parenchymal findings. Subsequently, an echocardiogram was performed at rest and with exercise to screen for pulmonary hypertension. The echocardiogram showed concentric left ventricular hypertrophy, normal left atrial size, mild elevation of pulmonary pressures with tricuspid regurgitation peak velocity of $2.9 \mathrm{~m} \cdot \mathrm{s}^{-1}$ during peak exertion, and normal right ventricular size and function. Trans-oesophageal echocardiogram with injection of agitated saline was performed to further evaluate her unexplained hypoxaemia. This study revealed the late appearance (after six cardiac cycles) of a large number of bubbles entering the left atrium through the coronary sinus, consistent with intrapulmonary shunting. Finally, a computed tomography (CT) angiogram of the lungs was performed and did not reveal a large arteriovenous malformation. Therefore, it was suspected that the patient had HPS.

Because HPS was suspected, we evaluated for underlying liver disease. An abdominal ultrasound revealed hepatic parenchymal disease with portal vein enlargement and retrograde flow consistent with portal hypertension. Laboratory analysis showed elevated direct and indirect bilirubin, elevated hepatic transaminases and alkaline phosphatase, and low serum albumin. Her prothrombin time and creatinine were within normal limits. Serologic evaluation revealed a ferritin level of 8,350 ng (normal 16-150 ng), with normal ceruloplasmin and negative studies for viral and autoimmune hepatitis.

The second patient was a 26-yr-old female with haemoglobin SS disease and history of long-term transfusion, typically in the setting of acute crisis with an associated acute decrease in haemoglobin $\left(<5 \mathrm{~g} \cdot \mathrm{dL}^{-1}\right)$. She presented with exertional hypoxaemia but had minimal exertional dyspnoea. On ambulation, her oxygen saturation decreased from $95 \%$ to $81 \%$. Her examination revealed icteric sclera and mild abdominal distension concerning for ascites. Her chest radiograph was normal, a contrasted CT scan did not reveal any evidence of pulmonary embolism or arteriovenous malformation, and her resting alveolar-arterial gradient was $22 \mathrm{mmHg}$. Transthoracic echocardiography with injection of agitated saline revealed evidence of shunting, but could not distinguish intracardiac versus intrapulmonary shunting. She then underwent right heart catheterisation. Pulmonary arterial pressures were normal, but the transhepatic gradient was elevated at $11 \mathrm{mmHg}$. During catheterisation, intracardiac echocardiography with injection of agitated saline showed the return of a large number of bubbles into the left atrium through the coronary sinus, diagnostic of intrapulmonary shunting and suggestive of HPS. Evaluation of liver function revealed chronically elevated transaminases, elevated conjugated bilirubin, low serum albumin and elevated prothrombin time. Her serum ferritin level was $594 \mathrm{ng}$, and other common causes of liver disease were evaluated and not present.

In conclusion, we report two patients with intrapulmonary shunting in the presence of hepatic dysfunction and chronic iron overload. While neither of our patients had liver biopsy to confirm the presence of cirrhosis (the test was refused in each case), both had laboratory results and examinations consistent with cirrhosis. As other causes of cirrhosis were excluded and both patients had significant iron overload, we attributed their liver disease to iron overload. Both patients had clear evidence of intrapulmonary shunting that was consistent with HPS. Therefore, in this clinical context, we have concluded that our patients developed HPS secondary to iron overload-induced 
cirrhosis. Both patients have been started on iron chelation and have been clinically stable in the months since their diagnosis.

Our findings are significant for several reasons. First, we do not believe that HPS with cirrhosis secondary to recurrent transfusions in patients with sickle cell anaemia has been previously described. Secondly, given the prevalence of iron overload-induced cirrhosis in patients with sickle cell anaesthesia, as well as the prevalence of HPS in patients with cirrhosis, there are likely to be other undiagnosed cases of HPS in this population. However, the decrease in nitric oxide availability in patients with sickle cell anaesthesia may have a protective effect against the formation of HPS, since elevated nitric oxide levels have been associated with the development of HPS [10]. Thirdly, HPS is not part of the current differential diagnosis of unexplained chronic hypoxaemia in patients with sickle cell anaesthesia. While conditions such as pulmonary venous hypertension, pulmonary arterial hypertension, chronic pulmonary embolism and interstitial lung disease should be first considered, we believe that HPS should also be considered in patients with chronic hypoxaemia. Finally, our report underscores the importance of new therapeutic options that are available to treat chronic iron overload.

\section{Daniel Grinnan*, Alice Herlihy* and Wally R. Smith ${ }^{\#}$}

*Dept of Pulmonary and Critical Care, and "Dept of Internal Medicine, Virginia Commonwealth University Health System, Richmond, VA, USA.

Correspondence: D. Grinnan, Dept of Pulmonary and Critical Care, Virginia Commonwealth University Health System, POB 980050, Richmond, VA 23298, USA. E-mail: dcgrinnan@ vcu.edu
Statement of Interest: None declared.

\section{REFERENCES}

1 Brittenham GM. Iron-chelating therapy for transfusional iron overload. N Engl J Med 2011; 364: 146-156.

2 Adams RJ, McKie VC, Hsu L, et al. Prevention of a first stroke by transfusions in children with sickle cell anemia and abnormal results on transcranial Doppler ultrasonography. $N$ Engl J Med 1998; 339: 5-11.

3 Adams RJ, Brambilla D. Discontinuing prophylactic transfusions used to prevent stroke in sickle cell disease. N Engl J Med 2005; 353: 2769-2778.

4 Adamkiewicz TV, Abboud MR, Paley C, et al. Serum ferritin level changes in children with sickle cell disease on chronic blood transfusion are nonlinear and are associated with iron load and liver injury. Blood 2009; 114: 4632-4638.

5 Inati A, Mussalam KM, Wood JC, et al. Iron overload indices rise linearly with transfusion rate in patients with sickle cell disease. Blood 2010; 115: 2980-2981.

6 Solomon LR. Iron-chelating therapy for transfusional iron overload. N Engl J Med 2011; 364: 1475-1476.

7 Schenk P, Fuhrmann V, Madl C, et al. Hepatopulmonary syndrome: prevalence and predictive value of various cut offs for arterial oxygenation and their clinical consequences. Gut 2002; 51: 853-859.

8 Rodriguez-Roisin R, Krowka M. Hepatopulmonary syndrome: a liver induced lung vascular disorder. N Engl J Med 2008; 358: 2378-2387.

9 Vig R, Machado R. Pulmonary complications of hemoglobinopathies. Chest 2010; 138: 973-983.

10 Degano B, Mittaine M, Herve P, et al. Nitric oxide production by the alveolar compartment of the lungs in cirrhotic patients. Eur Respir J 2009; 34: 138-144.

\section{A breathtaking reverse bungee jump}

\section{To the Editors:}

Bungee jumping became popular in the early 1980s. Owing to the prevailing professionalism in the field, accidents are extremely rare. However, occasional medical complications do occur. They are mostly ophthalmologic, but spinal and vascular lesions have been reported [1]. Respiratory complications have also been described (one case of pulmonary haemorrhage [2] and one case of bilateral pneumothoraces in a patient at risk [3]). Reverse bungee jumping is an increasingly widespread fairground attraction during which a one- or twoperson passenger cradle is hurled vertically up to $80 \mathrm{~m}$ into the air by the sudden release of previously stretched elastic ropes (fig. 1). Exceptional accidents have been reported (one case of subdural haematoma [4] and one case of pneumothorax [5]). We describe a case of acute hemidiaphragmatic elevation with diaphragm weakness possibly linked to this activity.

A 52-yr-old office worker $(175 \mathrm{~cm} ; 97 \mathrm{~kg}$; body mass index $31.7 \mathrm{~kg} \cdot \mathrm{m}^{-2}$ ) was referred to our outpatient clinic in May 2006 to investigate recent-onset exercise dyspnoea (self-characterised as "hindering usual acrobatic dance performances"). The patient also reported mild orthopnoea. He did not smoke and had no history of chronic disease, previous surgery or traumatism. Clinical examination was unremarkable. Chest radiography showed an elevated right hemidiaphragm (fig. 2a) capped by passive atelectasis. Thoracic computed tomography did not show other abnormalities. Blood chemistry and blood cell counts were normal. Vital capacity was $87 \%$ predicted, falling to $73 \%$ pred in the supine position. Sniff nasal inspiratory pressure was $57 \%$ pred $\left(64 \mathrm{cmH}_{2} \mathrm{O}\right)$. Cervical magnetic stimulation of the phrenic nerves showed electromyographic responses with normal latencies on both sides (right: $6 \mathrm{~ms}$; left: $6.3 \mathrm{~ms}$; expected values between 5.5 and $6.5 \mathrm{~ms}$ ) but diminished amplitude on the right side (right-toleft ratio 0.5$)$. The corresponding diaphragm twitch oesophageal pressure was low, at $4.7 \mathrm{cmH}_{2} \mathrm{O}$ (expected value $>11 \mathrm{cmH}_{2} \mathrm{O}$ ), and so was the twitch transdiaphragmatic pressure, at $10.9 \mathrm{cmH}_{2} \mathrm{O}$ (expected value $>20 \mathrm{cmH}_{2} \mathrm{O}$ ). 